\title{
Substrate effect on the magnetic behavior of manganite films
}

\author{
L. B. Steren, ${ }^{\text {a) }}$ M. Sirena, and J. Guimpel ${ }^{\text {a) }}$ \\ Centro Atómico Bariloche-Instituto Balseiro, Avda. Bustillo 9.5, (8400) S.C. Bariloche, Argentina
}

The film thickness $(t)$ dependence of the magnetic properties of $\mathrm{La}_{0.6} \mathrm{Sr}_{0.4} \mathrm{MnO}_{3}$ (LSMO) films grown on (001) $\mathrm{MgO}$ and (001) $\mathrm{SrTiO}_{3}$ substrates has been studied. Hysteresis loops measured at low temperature show a smooth increase of the retentivity accompanied by a decrease of the coercitive field as the film thickness increases. The increase of coercitivity with decreasing $t$ can be interpreted in terms of a change in the domain structure of the films mainly due to an augmentation of domain pinning defects. The magnetic anisotropy has been measured using ferromagnetic resonance (FMR). A volume (VA) and a surface (SA) anisotropy contribution have been deduced from FMR angular dependence studies for both series of samples. In the LSMO films grown on $\mathrm{MgO}$ a VA component that corresponds to an easy-axis perpendicular to the plane of the films has been found while in contrast, the LSMO films grown on $\mathrm{SrTiO}_{3}$ present an easy-plane anisotropy. The SA is positive for both series favoring a perpendicular magnetic anisotropy. The measured magnetic anisotropy has been assigned to substrate-induced effects. (C) 2000 American Institute of Physics. [S0021-8979(00)55708-9]

The "colossal" magnetoresistance (CMR) effect observed in doped manganite materials ${ }^{1}$ have received much attention in the last years due to its potential applications in magnetic devices. In contrast to the "giant" magnetoresistance observed in metallic nanostructures, ${ }^{2}$ the CMR is an intrinsic effect. The crucial role of the crystal structure and lattice distortions in the magnetic and transport properties of these materials has been investigated by Goodenough ${ }^{3}$ and co-workers. Experimental studies on the effect of hydrostatic pressure and cations substitution on the magnetic order and metal-insulator transition of bulk compounds have been reported in several papers. ${ }^{4,5}$ In films, substrates impose constraints that influence different physical properties like the magnetoresistance, the Curie temperature and the magnetic anisotropy. In a recent theoretical work, Millis and co-workers ${ }^{6}$ outlined the importance of biaxial strains and uniform compression pressure in the ferromagnetic ordering of manganate oxides and discussed possible effects on the transport properties of thin films. Zhang et al. ${ }^{7}$ and Ju et al. ${ }^{8}$ showed the effect of substrate-induced strains on the transport and magnetoresistive properties of manganite films. Recent investigations ${ }^{9,10}$ have reported a decoupling of the ferromagnetic order and the metal-insulator transition which depends on the thickness of the films. Suzuki et al. ${ }^{11}$ compared the magnetic anisotropy of $\mathrm{La}_{0.7} \mathrm{Sr}_{0.3} \mathrm{MnO}_{3}$ single crystal and films by magnetization and torque measurements.

In this article we present a systematic study of film thickness dependence of the magnetic behavior of manganite films deposited on different substrates. Our results were obtained from magnetization and ferromagnetic resonance (FMR) measurements.

Two series of $\mathrm{La}_{0.6} \mathrm{Sr}_{0.4} \mathrm{MnO}_{3}$ films were grown by dc sputtering on (100) $\mathrm{MgO}$ (LSMO-M) and (100) $\mathrm{SrTiO}_{3}$ (LSMO-S) substrates. The thickness $t$ of the samples was

\footnotetext{
a) Also at Consejo Nacional de Investigaciones Cientificas y Técnicas, Argentina.
}

varyed from $5 \mathrm{~nm}$ to $500 \mathrm{~nm}$. The EDAX analysis performed on the films showed that the films composition is that of target $\left(\mathrm{La}_{1-x} \mathrm{Sr}_{x} \mathrm{MnO}_{3} \quad x=0.4\right)$, within the experimental resolution (10\%). The x-ray diffraction patterns for the films only show LSMO pseudocubic $[001]_{c}$ and substrate related diffraction peaks, indicating a good texturation of the samples. The rocking curve width is found to be the same for all diffraction orders, indicating a mosaic spread origin. This width has a value of around $0.8^{\circ}$ for the LSMO-M films and $0.1^{\circ}$ for the LSMO-S films. For $t$ thinner than $50 \mathrm{~nm}$ this value increases, fact that could be due to thickness broadening of the diffraction peaks. The lattice parameter of the films was determined indexing in the pseudocubic structure. For the LSMO-M films $a_{c}=0.3882(4) \mathrm{nm}$ with negligible thickness dependence. For the LSMO-S films a smooth increase with thickness from $a_{c}=0.384 \mathrm{~nm}$ to $a_{c}=0.385 \mathrm{~nm}$ is observed. These structural results suggest that the growth mode of the two series of films is different. For the severely lattice mismatched LSMO-M system ( 9\%), we interpret the thickness independent lattice parameter and the increased rocking curve width as originating in a film which has relaxed the stresses in an "accommodation layer" of a few nanometers, probably through structural defects like dislocations or point defects. For the LSMO-S series, on the contrary, the thickness dependence of the lattice parameter and the smaller mosaic spread indicate the possibility of epitaxial growth. In effect, if epitaxial growth were present, the expansive biaxial stress introduced by the $\mathrm{SrTiO}_{3}$ substrate would produce a thickness dependent out-of-plane contraction, as observed. Preliminary results on pole figure x-ray diffraction show that the growth is indeed epitaxial both for $\mathrm{SrTiO}_{3}$ and $\mathrm{MgO}$ substrates, the later one surprisingly in spite of the large mismatch. These results will be published elsewhere. $^{12}$

Measurements of magnetization were performed using a SQUID magnetometer with the magnetic field applied in the plane of the films and normal to them. All the films present a 


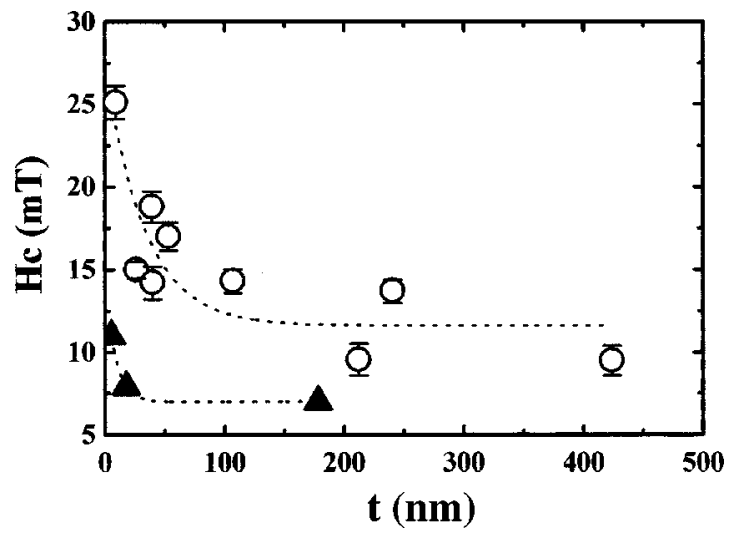

FIG. 1. Thickness dependence of the coercive field in $\mathrm{La}_{0.6} \mathrm{Sr}_{0.4} \mathrm{MnO}_{3}$ films. Open symbols correspond to the LSMO-M films and solid symbols to the LSMO-S ones. Solid lines are to guide the eye.

paramagnetic-ferromagnetic transition, that has been observed in remanent magnetization $\left(M_{\mathrm{REM}}\right)$ vs temperature curves. The onset of $M_{\mathrm{REM}}$ is a marker of the magnetic transition. The Curie temperature $\left(T_{C}\right)$ decreases from $238 \mathrm{~K}$ to $210 \mathrm{~K}$ when the film thickness decreases from $425 \mathrm{~nm}$ to 24 $\mathrm{nm}$ for the LSMO-M films and from $200 \mathrm{~K}$ to $188 \mathrm{~K}$ in the same thickness range for the LSMO-S films. The difference found in the ordering temperature of both systems can be attributed to the different strains induced by $\mathrm{MgO}$ and $\mathrm{SrTiO}_{3}$ substrates, respectively. However, all the measured values are lower than that of the bulk compound $(374 \mathrm{~K})$ evidencing a non-negligible effect of the substrates in both cases. $^{10}$

Different parameters of the hysteresis loops are thickness dependent but the saturation magnetization, $M_{s}$, measured at low temperature corresponds to the expected value $\left(M_{s}=545 \mathrm{emu} / \mathrm{cm}^{3}\right)$ in all the samples. A smooth increase of the retentivity $\left(M_{\mathrm{REM}} / M_{s}\right)$ together with a decrease of the coercitive fields as the thickness of the films increases is observed in $50 \mathrm{~K}$ hysteresis loops of both LSMO-M and LSMO-S films. This dependence is more noticeable in the former series. The retentivity increases exponentially with $t$, saturating above $100 \mathrm{~nm}$ at $0.5 M_{s}$ in both series of samples. The saturation value can be interpreted in terms of biaxial anisotropy axis or random oriented anisotropy axis. ${ }^{13}$ The reduction of $M_{\mathrm{REM}} / M_{s}$ below $100 \mathrm{~nm}$ could be explained by a change of the magnetic anisotropy of the films, as will be discussed below. The change of coercitivity with the film thickness can be attributed to a consequent change in the domain structure of the samples. Higher coercitive fields could be attributed to higher in-plane anisotropy or an augmentation of domain pinning defects (Fig. 1). As we already mentioned, a more disordered structure is expected in the thinner films where the lattice mismatch with the substrate have to be overcome and so the second argument seems more plausible.

Room temperature loops (far above $T_{C}$ ) present a reversible behavior for $t_{r}>100 \mathrm{~nm}$ and very small hysteresis (retentivity $\sim 0.10$ and coercive fields $\sim 2 \mathrm{mT}$ ) for films thinner than $t_{r}$. The reversible component can be fitted by a Brillouin function but the calculated moments are much bigger

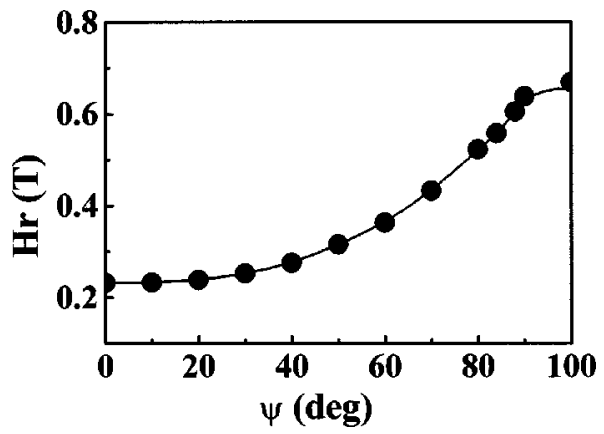

FIG. 2. Resonance fields for the LSMO-M, $t=53.2 \mathrm{~nm}$ film, measured at $150 \mathrm{~K}$. Solid line is a guide to the eyes.

than the ones corresponding to paramagnetic manganese ions. The hysteresis measured above $T_{C}$ may indicate that the samples are not magnetically homogeneus; it suggests the existence of short-range ordered regions, already observed by neutron diffraction in bulk materials. ${ }^{14}$ The disordered regions would be mainly located at the substrate interface, representing a small fraction of sample in thick films.

The hysteresis loops measured with the magnetic field applied perpendicular to the films show saturation fields close to the magnetostatic one $\left(4 \pi M_{s}\right)$ for the thick films. However, a progressive departure from this value is observed with decreasing thickness, indicating the effects of other sources of anisotropy. FMR measurements were performed to study with more detail the magnetic anisotropy of the samples. These measurements were carried out at $X$ band $(9.3 \mathrm{GHz})$ in a BRUKER spectrometer, in the $100-350 \mathrm{~K}$ temperature range. We studied the angular dependence of the FMR spectrum as the applied dc field is rotated from the plane of the films to its perpendicular direction. The resonance fields as a function of the angle between field and film plane, $\Psi$, are shown in Fig. 2. To quantify the anisotropy constants, the perpendicular and parallel resonance fields are fitted with the resonance conditions, ${ }^{15}$

$$
\begin{aligned}
& {[\omega / \gamma]^{2}=H_{\|}\left(H_{\|}+4 \pi M_{s}-H a\right),} \\
& \omega / \gamma=H_{\perp}-\left(4 \pi M_{s}-H a_{1}\right),
\end{aligned}
$$

where $\omega$ is the microwave frequency, $\gamma$ is the gyromagnetic ratio, $H_{\|}$and $H_{\perp}$ are the resonance fields parallel and perpendicular to the plane of the films, respectively. The anisotropy fields in Eqs. (1) and (2) are defined by $H a=H a_{1}$ $+H a_{2}, H a_{1}=2 K_{1} / M$, and $H a_{2}=4 K_{2} / M$, being $K_{1}$ and $K_{2}$ the effective first-order and second-order anisotropy constants. The term $4 \pi M_{s}$ corresponds to the shape anisotropy.

The total magnetic anisotropy can be written as ${ }^{16}$

$$
K=K_{V}+1 / t 2 K_{S},
$$

where $K_{V}$ is the volume contribution (VA) and $K_{S}$ the surface-induced (SA) one. The prefactor 2 accounts for the two interfaces of the films. An average value of $K_{s}$ will be estimated for our samples due to the impossibility of distinguish between the anisotropy of each interface.

The calculated values of $H a_{1}$ and $H a_{2}$ were plotted in Fig. 3 as a function of $1 / t$ in order to analyze our results in terms of volume and surface contributions. It can be seen 


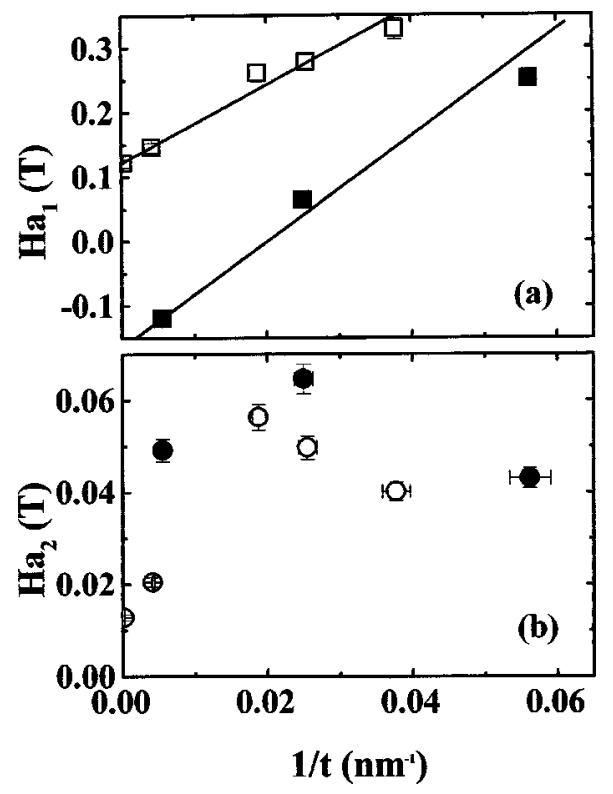

FIG. 3. Anisotropy fields, (a) $H a_{1}$ and (b) $H a_{2}$, plotted as a function of the reciprocal of the film thickness, $t$. The open symbols correspond to the LSMO-M films and solid symbols to the LSMO-S ones.

that $H a_{1}$ varies linearly with $1 / t$ in both series but no clear dependence of $\mathrm{Ha}_{2}$ on the film thickness is observed in either of them. From the slope of the lines $H a_{1}$ vs $1 / t, K_{S}$ have been determined for both series finding $K_{S}($ LSMO-M $)$ $=+0.66 \mathrm{erg} / \mathrm{cm}^{2}$ and $K_{S}(\mathrm{LSMO}-\mathrm{S})=+0.89 \mathrm{erg} / \mathrm{cm}^{2}$, respectively. The SA is positive for the two series, thus favoring a perpendicular to the film anisotropy. Roughness and interdiffussion at the interfaces of the films modify the magnetostatic fields, introducing a SA, always positive but small $\left(\sim 0.1 \mathrm{erg} / \mathrm{cm}^{2}\right)$ in general. ${ }^{16}$ The magnetoelastic energy (ME) also contributes to the SA when the film growth is not completely coherent. An important contribution from structural disorder could be particularly expected for the LSMO-M series due to the large lattice mismatch between the manganate films and the $\mathrm{MgO}$. In spite of this fact, the SA is bigger for the LSMO-S series, suggesting that the ME contribution plays a significant role in it.

The extrapolated $K_{V}$ values are $K_{V}(\mathrm{LSMO}-\mathrm{M})=+2.6$ $\times 10^{5} \mathrm{erg} / \mathrm{cm}^{3}$ and $K_{V}($ LSMO-S $)=-3.6 \times 10^{5} \mathrm{erg} / \mathrm{cm}^{3}$. The magnitude of the volume anisotropy is similar for both series but of opposite sign. The first one corresponds to an easy uniaxial axis, parallel to the film normal while the second one is associated to an easy-plane, in the plane of the films. The volume contribution may arise from magnetocrystalline or stresses anisotropy. In single crystals the main contribution to the magnetic anisotropy is the magnetocrystalline (MCA) one. Suzuki et al. ${ }^{11}$ observed a twofold symmetry in $\mathrm{La}_{0.7} \mathrm{Sr}_{0.3} \mathrm{MnO}_{3}$ single crystals and attributed this result to a rhombohedral MCA with $K=1.8 \times 10^{4} \mathrm{erg} / \mathrm{cm}^{3}$. In our case, the measured anisotropy is one order of magnitude bigger than the MCA and has a characteristic sign depending on the substrate of the films, facts that lead to the assignment of this anisotropy to be originated in substrate-induced strains and structural defects. The anisotropy constants estimated for our films are comparable to those found by O'Donnell et al. ${ }^{17}$ and Nath et al. ${ }^{18}$ in other manganite films.

In conclusion, we have studied the thickness dependence of the magnetic behavior of manganite films grown on different substrates. We have found a general behavior for some parameters; (a) the ferromagnetic ordering temperature depresses, (b) the coercitive field increases, and (c) the remanence of the samples decreases with decreasing film thickness. On the other hand, the magnetic anisotropy is very sensitive to the substrate of the samples changing from an easy-plane to an easy-axis perpendicular to the film symmetry, evidencing its structural origin (magnetoelastic and disorder).

The authors wish to thank J.C. Perez, R. Benavidez, and J. Azcarate for technical support. This work was partially supported by the CONICET (Grant PEI No. 0123/97), by the ANPCYT (Grant PICT 97 No. 03-00052-01025).

${ }^{1}$ R. von Helmholt, J. Wecker, B. Holzapfel, L. Schultz, and K. Samwer, Phys. Rev. Lett. 71, 2331 (1993).

${ }^{2}$ M. Baibich, J. Broto, A. Fert, F. Nguyen Van Dau, F. Petroff, P. Etienne, G. Creuzet, A. Friederich, and J. Chazeles, Phys. Rev. Lett. 61, 2472 (1988).

${ }^{3}$ J. Goodenough, Interscience Monographs on Chemistry (Wiley, New York, 1963), Vol. I.

${ }^{4}$ J. Fontcuberta, V. Laukhin, and X. Obradors, Appl. Phys. Lett. 72, 2607 (1998).

${ }^{5}$ L. Rodriguez-Martinez and J. P. Attfield, Phys. Rev. B 54, R15622 (1996).

${ }^{6}$ A. Millis, T. Darling, and A. Migliori, J. Appl. Phys. 83, 1588 (1998).

${ }^{7}$ W. Zhang, X. Wang, M. Elliott, and I. Boyd, Phys. Rev. B 58, 14143 (1998).

${ }^{8}$ H. Ju, K. Krishnan, and D. Lederman, J. Appl. Phys. 83, 7073 (1998).

${ }^{9}$ J. Aarts, S. Freisem, R. Hendrikx, and H. Zandbergen, Appl. Phys. Lett. 72, 2975 (1998).

${ }^{10}$ L. B. Steren, M. Sirena, and J. Guimpel, J. Magn. Magn. Mater. (in press).

${ }^{11}$ Y. Suzuki, H. Hwang, S-W. Cheong, T. Siegrist, R. van Dover, A. Asamitsu, and Y. Tokura, J. Appl. Phys. 83, 7064 (1998).

${ }^{12}$ M. Sirena, L. B. Steren, J. Guimpel, O. Durand, and E. Sanchez (to be published).

${ }^{13}$ R. Bozorth, Ferromagnetism (Van Nostrand, New York, 1953).

${ }^{14}$ J. M. De Teresa, R. Ibarra, J. Blasco, J. Garcia, C. Marquina, P. Algarabel, Z. Arnold, K. Kamenev, C. Ritter, and R. Von Helmolt, Phys. Rev. B 54, 1187 (1996).

${ }^{15}$ C. Chappert, K. LeDang, P. Beauvillain, H. Hunderquint, and D. Renard, Phys. Rev. B 34, 3192 (1986).

${ }^{16}$ W. J. de Jonge, P. Bloemen, and F. den Broeder, Ultrathin Magnetic Structures I, edited by J. Bland and B. Heinrich (Springer-Verlag, Berlin, 1994), p. 65

${ }^{17}$ J. O'Donnell, H. Rzchowski, J. Eckstein, and I. Bozovic, Appl. Phys. Lett. 72, 1775 (1998)

${ }^{18}$ T. Nath, R. Rao, D. Lavric, C. Eom, L. Wu, and F. Tsui, Appl. Phys. Lett. 74, 1615 (1999) 